Service social

\title{
Mouvement populaire et intervention communautaire de 1960 à nos jours, Continuités et ruptures, par Louis Favreau, Montréal, le Centre de formation populaire et les Éditions du Fleuve, Alternatives, 1989, 307 pages.
}

\section{René Falardeau}

Volume 38, numéro 2-3, 1989

Les politiques sociales

URI : https://id.erudit.org/iderudit/706447ar

DOI : https://doi.org/10.7202/706447ar

Aller au sommaire du numéro

Éditeur(s)

École de service social de l'Université Laval

ISSN

1708-1734 (numérique)

Découvrir la revue

Citer ce compte rendu

Falardeau, R. (1989). Compte rendu de [Mouvement populaire et intervention communautaire de 1960 à nos jours, Continuités et ruptures, par Louis Favreau, Montréal, le Centre de formation populaire et les Éditions du Fleuve, Alternatives, 1989, 307 pages.] Service social, 38(2-3), 321-322.

https://doi.org/10.7202/706447ar d'utilisation que vous pouvez consulter en ligne. 
Les pistes d'intervention proposées sont des plus intéressantes. Cependant, il aurait été très avantageux que les auteurs explorent plus à fond les modèles complémentaires de prise en charge de ces clientèles. Pour que ces jeunes traversent ces périodes de crise avec le moins de séquelles possible, la multidisciplinarité s'impose aux intervenants des milieux social, communautaire, médical et de l'éducation.

À qui incombe la protection de l'enfance maltraitée ? Ne serait-ce pas une responsabilité collective?

Marcel BONNEAU

Direction de la Protection de la jeunesse,

Sherbrooke.

Mouvement populaire et intervention communautaire de 1960 à nos jours, Continuités et ruptures, par Louis FAVREAU, Montréal, le Centre de formation populaire et les Éditions du Fleuve, Alternatives, 1989, 307 pages.

Le mouvement populaire et communautaire s'engage-t-il dans une période de déclin ou de mutation culturelle et sociale ? Voilà la question centrale qui est à l'origine d'une importante enquête qui a débuté en 1985 et qui demeure d'actualité en 1989.

Pour répondre à cette question, l'auteur nous invite à faire un peu $d$ 'histoire en abordant la dimension historique du mouvement populaire et communautaire québécois et ce pour les vingt-cinq dernières années. L'itinéraire qui nous est suggéré nous amène de la naissance des premiers comités de citoyens de la décennie 60 , en passant par les belles années du militantisme, les années d'incertitudes, de tâtonnements, pour conclure sur la possible relance de ce mouvement.

Le troisième chapitre nous fait pénétrer et sentir le riche vécu de trois quartiers populaires montréalais : Hochelaga-Maisonneuve, Centre-Sud et La Petite Patrie. À travers I'analyse de plusieurs groupes populaires, I'auteur cherche à dégager des constantes concernant le démarrage, la survie et la consolidation de ces groupes. Ces quartiers ont subi et continuent de vivre une mutation sociale très importante; alors qu'hier la culture de pauvreté était très présente, aujourd'hui elle se fait envahir par une nouvelle mentalité qui peut se caractériser par une capacité de transformer les problèmes en projets.

Ce volume aborde aussi avec beaucoup de justesse et d'honnêteté la difficile question de l'implication du mouvement populaire et communautaire dans l'économie et plus précisément dans la création d'emplois et d'entreprises. La récession du début des années ' 80 a bouleversé bien des valeurs. Le mouvement populaire a un nouveau visage. D'un côté, l'implication des militants et militantes sur le terrain de l'économie est une réalité bien présente; de l'autre côté, les groupes de services et de pression demeurent et continuent d'exercer une forme de pouvoir non négligeable. Dans ce chapitre, 
le lecteur se laissera sans aucun doute interpeller par la problématique de l'économie communautaire qui continue de soulever de nombreux débats.

Le cinquième chapitre traite de l'engagement du mouvement populaire et communautaire dans trois expériences d'actions politiques vécues précisément dans la région de Montréal. II est notamment question du Front d'action politique (FRAP), du courant politique " marxisme-léninisme " qui a marqué la gauche des années '70, et du cheminement qui a amené le Rassemblement des citoyens de Montréal (RCM) à la prise du pouvoir. À travers ces trois expériences, on peut souligner entre autres choses la transformation radicale du militantisme. Alors que les années '60-'70 étaient caractérisées par un engagement total à la cause, on retrouve partout, dans les années ' 80 , cette volonté de vivre autrement maintenant.

Le chapitre suivant aborde l'épineuse question du rôle de l'État dans le financement des groupes populaires et communautaires. Un changement marqué dans la façon de voir l'intervention communautaire en C.L.S.C. s'est amorcé et nous amène à un tournant important qui modifiera les rapports entre le secteur communautaire et le secteur public. Les formes de cohabitation et de collaboration ne seront plus les mêmes. Un financement adéquat est maintenant perçu comme un prérequis essentiel à la liberté d'orientation, d'intervention et de gestion du secteur communautaire.

La contribution originale de l'auteur consiste donc en une étude fort bien détaillée qui, à l'image d'un album-photos, nous permet de mieux saisir une époque significative du mouvement populaire et communautaire. II nous amène sur la piste d'un tournant majeur qui permet de récuser l'idée du déclin de ce mouvement et propose une vision nouvelle marquée par une transformation sociale et culturelle, mais aussi, par les continuités et les ruptures.

René FaLARDEAU

Association pour l'intégration sociale, Québec.

Psychologie du travail et nouveaux milieux de travail, par Alain LAROQUE, Yvan BordeleaU, René Boulard, Bruno Fabi, Viateur Larouche et Alain Rondeau (comité d'édition), Sillery, Presses de I'Université du Québec, 1987, 753 pages.

Sous l'égide de son Chapitre canadien, l'Association de psychologie du travail de langue française a tenu à Montréal les 5, 6 et 7 mai 1986, son quatrième Congrès sur le thème $P$ sychologie du travail et nouveaux milieux de travail. Ce volume présente intégralement les 62 communications qui y furent prononcées par des psychologues du travail, spécialistes en relations industrielles et autres disciplines connexes. Les participants sont venus du Canada (Québec), des États-Unis, de la France, de la Belgique, de la Pologne, de la Turquie et de I'Argentine. 